\title{
A Feasibility Study of a One-to-One Mindfulness-Based Intervention for Improving Mood in Stroke Survivors
}

\author{
Wendy Wrapson $^{1} \cdot$ Marlies Dorrestein ${ }^{1} \cdot$ Jill Wrapson ${ }^{1} \cdot$ Alice Theadom $^{1} \cdot$ Nicola M. Kayes ${ }^{1} \cdot$ Deborah L. Snell $^{2}$. \\ Sandy Rutherford ${ }^{1} \cdot$ Maree Roche $^{3} \cdot$ Duncan R. Babbage $^{1} \cdot$ Steve Taylor $^{1} \cdot$ Richard J. Siegert $^{1}$ (D)
}

Accepted: 8 December 2020 / Published online: 8 January 2021

(C) The Author(s) 2021

\begin{abstract}
Objectives Mindfulness is an evidence-based treatment for depression but has never been rigorously tested with stroke survivors with depression. This feasibility study examined several issues relevant to a potential trial of a mindfulness-based intervention (MBI) for improving mood after stroke.

Methods In 2017-2019 in New Zealand, we recruited 20 stroke survivors with low mood to undergo a 6-week, one-on-one MBI course delivered by an occupational therapist experienced in MBIs. Pre, post, and 4-week follow-up assessments were completed.

Results Fifteen participants completed all six sessions and a 4-week "booster" or top-up session. The 1-hour session duration was considered appropriate by participants and all enjoyed the face-to-face individualized format. Mean Beck Depression InventoryII scores improved by more than one standard deviation and this was maintained at follow-up. However, the baseline assessment package was too long for some participants due to the cognitive component. Three participants indicated feeling emotionally challenged by some of the practices. These effects were managed by the mindfulness facilitator by adjusting the practice, so participants maintained their sense of agency, well-being, and overall benefit from the program.

Conclusions MBI training delivered individually over six weekly sessions was acceptable to stroke survivors with 14/15 participants reporting improved mood. Three participants reported feeling emotionally challenged by some of the practices and we recommend MBIs for stroke survivors be provided by practitioners experienced in mindfulness, working with stroke, and trauma-informed therapy. It is important now to conduct rigorous randomized controlled trials to test the effectiveness and efficacy of MBIs for stroke survivors.
\end{abstract}

Keywords Mindfulness $\cdot$ Stroke $\cdot$ Anxiety $\cdot$ Depression $\cdot$ Feasibility

Almost a third of stroke survivors experience major depression in the 5 years after their stroke. Depression is associated with poorer functional outcomes (Anderson et al. 2004; Hackett and Pickles 2014; Taylor et al. 2006). Despite this, there is little evidence on the effective treatment of depression after stroke and there is an urgent need for trials of safe and easily delivered interventions for reducing depression and

Richard J. Siegert

richard.siegert@aut.ac.nz

1 Department of Psychology and Neuroscience, Faculty of Health and Environmental Sciences, Auckland University of Technology, Auckland, New Zealand

2 University of Otago, Christchurch, New Zealand

3 University of Waikato, Hamilton, New Zealand preventing relapse (Hackett et al. 2009; Hackett and Pickles 2014). There is almost no evidence on the effective management of anxiety after stroke despite its high prevalence (Burton et al. 2011; Cumming et al. 2016). A mindfulnessbased intervention (MBI) is typically a structured, selfmanagement program which has shown positive benefits across a range of physical and mental health conditions including improving mood and anxiety (de Abreu Costa et al. 2019; Thomas et al. 2020). In stroke, MBIs have demonstrated a positive trend across a range of psychological, physiological, and psychosocial outcomes including anxiety and depression, although sample sizes tend to be small and methodological quality is limited (Lawrence et al. 2013). MBIs appear relatively economical to deliver and can be undertaken in a variety of therapeutic contexts and locations; however, methodologically robust trials are needed to establish the 
effectiveness and acceptability of MBIs for stroke survivors (Lawrence et al. 2013; Siegert et al. 2015).

Before rigorous trials of MBIs for treating anxiety and/or depression in stroke survivors can take place, a number of important feasibility issues need addressing. For example, the standard mindfulness-based stress reduction (MBSR) program involves eight weekly sessions of two and a half hours length plus a one-day retreat (Alsubaiea et al. 2017). Integral to the MBSR program is that participants are expected to practice mindfulness exercises for periods of $45 \mathrm{~min}$ daily. This regimen could be challenging for stroke survivors who frequently have physical problems (e.g., hemiplegia, spasticity, chronic pain, fatigue), cognitive problems (e.g., reduced attention/concentration, executive dysfunction), and/or emotional problems (anxiety, depression). Hence, the issue of how training should be tailored for people after stroke in terms of the duration, frequency, mode of delivery, and content of sessions requires some exploration. In a systematic review of mindfulness interventions following stroke and transient ischemic attack, Lawrence et al. (2013) reported four studies with three using a group format and one delivered one-to-one. Three of these studies used MBSR, one used mindfulnessbased cognitive therapy (MBCT), and there were typically about eight weekly sessions, although 15 in the case of the one to one intervention. Actual session length varied from 1.75 to $2.5 \mathrm{~h}$ duration. In one of the few inpatient studies, Wang et al. (2019a) taught mindfulness in a stroke rehabilitation setting in two, weekly 90-min group sessions with individual daily practice for 2 weeks.

Another important issue is the possibility of unpleasant, adverse, or unwanted side effects from meditation. While mindfulness is generally regarded as a safe and non-intrusive practice, unpleasant, or distressing side-effects have occasionally been reported. Lazarus (1976) observed that meditation and relaxation practices were beneficial for many people but contraindicated for some and described a number of "meditation casualties" he had encountered clinically. These included several patients whose depressive affect had worsened despite meditation and one woman who had attempted suicide after a weekend course in Transcendental Meditation. In a recent review, Baer et al. (2019) concluded that only a small minority of studies has even monitored the presence of adverse events and serious adverse events and argued that this issue warrants attention. A recent systematic review that examined 83 studies noted the total prevalence of adverse events was $8.3 \%$ with anxiety and depression the most common (Farias et al. 2020). The authors cautioned that these frequently occurred in participants with no previous history of mental disorder and noted that there is no obvious way of identifying individuals most prone to such experiences.

Two studies have investigated the feasibility and acceptability of MBIs for stroke survivors. Jani et al. (2018) delivered a single two-hour MBSR "taster" session to 21 stroke survivors and 7 caregivers and reported generally positive feedback. However, participants suggested they would prefer shorter sessions and an initial orientation if embarking on a full course of MBSR. Wang et al. (2019b), using data from focus group discussions, reported that an intervention comprising mindfulness exercises recorded on DVD was acceptable to participants, although the authors noted that less than two-thirds of participants (24/38) were retained at postintervention follow-up. Neither of these feasibility studies with stroke survivors was focused on participants with depression and/or anxiety as assessed at baseline.

The aim of the present study was to examine a number of important feasibility issues relevant to providing a MBI to stroke survivors with low mood. We hypothesized that the stroke survivors would find completing the MBI to be an acceptable and beneficial experience.

\section{Methods}

\section{Participants}

Using purposive sampling methods, 43 participants from within the Auckland region in New Zealand were provided with detailed information about the study. Recruitment was via existing professional networks including stroke support organizations and rehabilitation providers, as well as advertisements in community newspapers and on public noticeboards. Advertising material informed potential participants that volunteers were sought for a feasibility study to find out whether a MBI could help with reducing anxiety and low mood after stroke.

The inclusion criteria were as follows: adults ( $>16$ years) who: (i) had experienced a stroke $>6$ months and $<10$ years ago; (ii) were experiencing low mood (Beck Depression Inventory II (BDI-II) score > 14) (Beck et al. 1996); and (iii) were able to give informed consent. Participants on antidepressive medication were included provided they were on a stable medication regime. These eligibility criteria were set to include people with persistent impairments and who were unlikely to be receiving ongoing funded rehabilitation services. The exclusion criteria included unstable co-morbid conditions, severe cognitive, and/or communication difficulties that would interfere with participation; a history of epileptic seizures or a first degree relative with epilepsy; current involvement in other psychological therapy; or unable to communicate in English or read simple written instructions. We excluded participants with a history of epileptic seizures or a first degree relative with epilepsy because of the unproven suggestion that in some people with a predisposition to epileptic seizures meditation might actually precipitate seizures (Jaseja 2006). This was admittedly a very cautious measure and we are aware of the growing evidence for the positive 
effects of meditation in people with epilepsy (Walker et al. 2010; Wood et al. 2017).

Those who expressed interest in taking part in the study and who indicated current presence of low mood completed the BDI-II (Beck et al. 1996) (by telephone, posting in a mailed copy, or personal visit by a researcher) to ensure they met the threshold for depression, the primary outcome variable. Twenty-two of the 43 participants provided with information about the study either did not meet the eligibility criteria (17), declined to participate (3), or were not contactable following the initial communication (2) (see Fig. 1). Of the 21 remaining, one participant withdrew after giving consent due to a lengthy hospitalization; another withdrew following completion of the baseline assessment and before completing any MBI sessions; and a third was found to be ineligible during the MBI when it was learnt they were also participating in a "yoga psychology" course unbeknownst to the research team. One participant who scored 16 on the BDI-II at the initial telephone screening, but only scored 8 at the baseline assessment 6 weeks later, was retained resulting in 18 participants who completed the baseline assessment protocol and started the MBI.

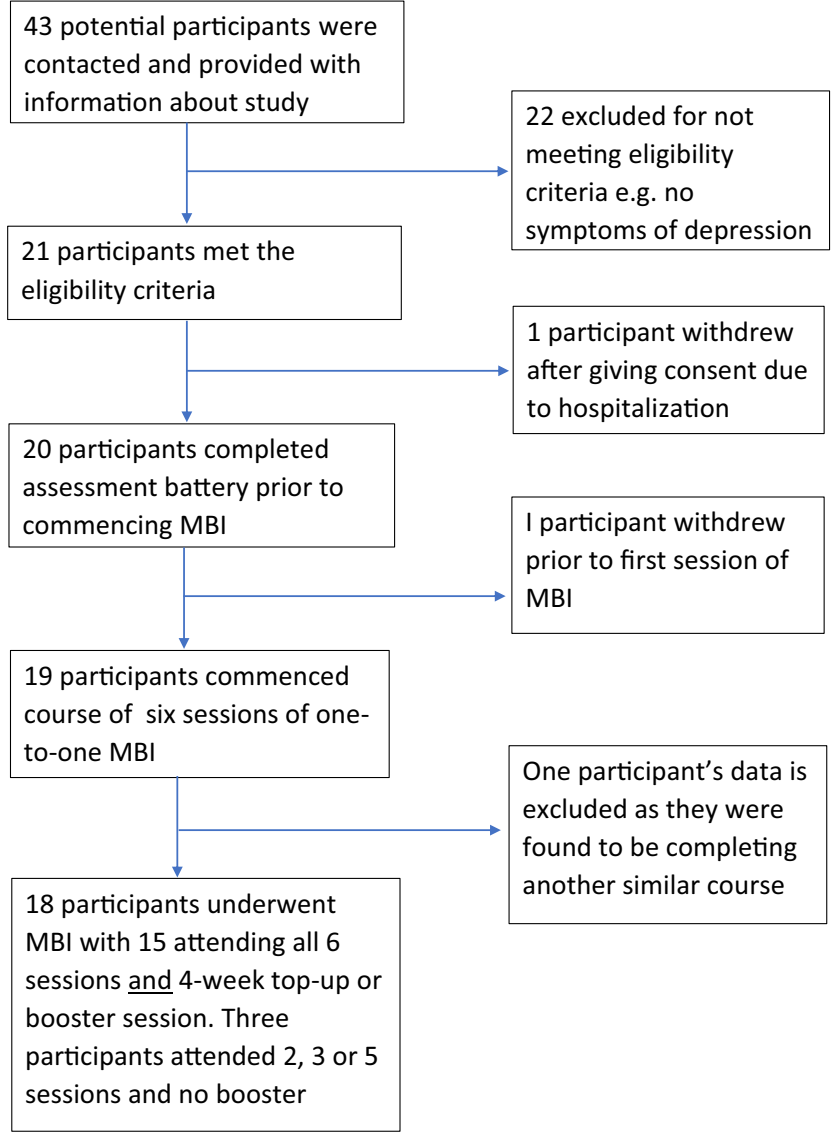

Fig. 1 Recruitment and retention

\section{Procedure}

The MBI was based on a MBSR program but modified to individual sessions of 60 min to offset any difficulties with participants' fatigue and concentration, and the course was shortened to 6 weeks to encourage completion of the intervention. We used individual sessions in participants' own homes for delivery of the proposed intervention, to ensure participants' impairments (e.g., fatigue, mobility, driving restrictions) were not a barrier to attendance. It was important to establish that the one-on-one delivery mode, in the absence of group support, was acceptable to participants.

The MBI was delivered by an occupational therapist (MD) with 25 years' personal practice of mindfulness, 2 years' experience as a mindfulness teacher, and who was supervised by a nationally recognized mindfulness trainer. Weekly sessions were scheduled with the therapist for six consecutive weeks, followed by a "top-up session" 4 weeks later. The MBI was specifically tailored for stroke survivors including simplified language and the use of repetition to help reinforce concepts. Due to the differences in impairment among participants, the therapist also tailored the content to address any specific physical or cognitive needs of individuals. For example, the "mindful movement" practice was incorporated into everyday activities such as walking, getting dressed, eating, or other activities that were meaningful for the participant. The week-byweek course structure and content are shown in Table 1 . Additional information regarding the mindfulness intervention is available in the completed Template for Intervention Description and Replication (TIDieR; Hoffmann et al. 2014) in the online Supplementary Material.

Information sheets on each session, together with a CD containing guided mindfulness exercises recorded by the therapist, were provided to participants so that they could practice what they had learnt each week in between sessions. A popular self-help book on mindfulness was also provided as a gift for taking part in the study, which was handed to participants at the end of the course.

Data collection took place in Auckland, New Zealand, between September 2017 and August 2019. A researcher initially met with participants to conduct a pre-intervention assessment. Depression and anxiety were measured using the BDI-II (Beck et al. 1996) and Hospital Anxiety and Depression Scale (Zigmond and Snaith 1983) respectively. The BDI-II was included to examine its feasibility as a primary outcome measure in a future trial.

Follow-up assessments were also conducted within 1 to 2 weeks of completion of the 6 -week course, and 1 week after the top-up session. During the first post-intervention assessment (and prior to the booster session), interviews were conducted with participants to seek their views on the MBI and whether they thought any changes should be made to the content and delivery should a full randomized controlled trial 
Table 1 Structure and content of mindfulness-based intervention

Structure for each session:

1. Welcome and rapport-building

2. Key reflective question on experience from previous week's practice

3. Practice (a different practice each week)

4. Reflection and discussion on experience and some theoretical underpinnings ("digging deeper") and hand out information sheet

5. Discussion and tips - exploring and documenting self-practice for the coming week
Week 1

Introduction and getting to

know each other

Main practice

Mindfulness of

body- -

breathing

anchor

Befriending the

breath

Digging deeper information:

Intro to course.

What is mindfulness?

Mindfulness in everyday life Week 4

Main practice:

Open heart-

loving

Kindness

practice

Digging deeper information:

Impact of

kindness

towards self

The benefit of recognizing simple pleasures

Follow-up week

Digging deeper Digging deeper information: information:

Importance of the body

Formal and informal

Introduction to mindful movement, e.g., walking or other daily activities

practice

Week 5

Main practice:

Mindfulness of feelings and thoughts

Relieving edges of frustration

Digging deeper information:

Recognizing the impact of thoughts

The benefit of acknowledging, or Follow-up in 4 weeks' time accepting

experience

Invite participant's reflection on experience

Check any questions or issues

Check which practice to repeat; brief review of the practices, practice, re-evaluate experience

Where to from here: ongoing self-practice resources, community resources

\section{Measures}

\section{Beck Depression Inventory-II}

The BDI-II measures severity of depression with 21 items scored from 0 to 3 . Total score can range from 0 to 63 with 0-13 representing minimal, $14-19$ mild, 20-28 moderate, and $\geq 29$ severe depression. The BDI-II manual reports high internal reliability for outpatients $(0.92)$ and college students $(0.93)$ and high test-retest (0.93) for outpatients tested a week apart (Beck et al. 1996).

\section{Hospital Anxiety and Depression Scale}

A brief measure of anxiety and depression with 14 items scored 0-3 and separate scales for anxiety ( 7 items) and depression (7 items) (Zigmond and Snaith 1983). Internal consistency is high for both anxiety (0.92) and depression $(0.88)$ in older (65-80 years old) people (Djukanovic et al. 2017).

\section{Stroke Specific Quality of Life Scale}

Comprises 49 items scored from 1 to 5 with total score ranging from 49 to 245 with higher scores representing higher quality of life. The Stroke Specific Quality of Life Scale (SSQOL) comprises 12 domains each comprising 3-6 items with Cronbach's alpha ranging from $0.81-0.94$ and test-retest reliability ranging from $0.65-0.99$ for individual domains (Muus et al. 2007; Williams et al. 1999).

\section{Modified Fatigue Impact Scale}

A brief measure of fatigue with 21 items scored from 0 to 4 with high scores representing high levels of fatigue. The fullscale score has high internal reliability (0.92) (Kos et al. 2005).

\section{Five Facet Mindfulness Questionnaire}

A measure of mindfulness with 39 items scored from 1 to 5 with five subscales, namely observing (8 items), describing (8), acting with awareness (8), nonjudging of inner experience (8), and nonreactivity to inner experience (7). Cronbach's alpha for the five subscales is typically high $(0.82-.90)$ (Gu et al. 2016).

\section{The Working Memory and Processing Speed Indices from the WAIS-IV}

be undertaken in the future. The results of these qualitative interviews are to be reported in a separate and forthcoming article. Participants received a $\$ 30$ fuel or supermarket voucher for completing each of the follow-up assessments and for the interview (\$90 in total).
The Working Memory Index (WMI) is a composite score based on the Arithmetic, Digit Span, and Letter-Number Sequencing subscales of the WAIS-IV. The WMI has high internal $(0.94)$ and test-retest $(0.88)$ reliability (Wechsler 
2008). The Processing Speed Index of the WAIS-IV is drawn from the Cancelation, Coding, and Symbol Search subscales and has high internal $(0.90)$ and test-retest $(0.87)$ reliability (Lichtenberger and Kaufman 2009).

\section{Advanced Clinical Solutions Word Choice Test}

This is a 50 -item forced word list that takes about $5 \mathrm{~min}$ and is widely used to assess performance validity. The Word Choice Test (WCT) has well-established classification accuracy (sensitivity and specificity) and validity (Bain and Soble 2019; Pearson 2009).

\section{Leeds Dependency Questionnaire}

Participants also completed an alcohol and drug dependency screening tool, the Leeds Dependency Questionnaire (LDQ), comprised of 10 items scored on a Likert scale from 0 (never) to 3 (nearly always). Scores can range from 0 to 30 with zero representing no dependence, 1-10 low/moderate, 11-20 moderate to high, and $\geq 21$ high dependence. The internal consistency is very high (0.93) (Kelly et al. 2010; Raistrick et al. 1994).

\section{Feasibility}

In reporting this study, we use the framework developed by Bowen et al. (2009) for conceptualizing feasibility studies. According to this framework, feasibility studies address eight general areas of focus, of which we examined five: (1) acceptability, (2) demand, (3) implementation, (4) expansion, and (5) limited-efficacy testing.

Acceptability focuses on how the people the intervention is designed to assist react to it. In our case, we aimed to determine the acceptability of the time commitment for weekly MBI sessions with daily practice and also to examine the possibility of unpleasant or adverse effects. Demand focuses on the consumer or client demand for the intervention by collecting data on actual use or uptake. In our study, we were interested to assess the feasibility of the recruitment strategy to yield adequate numbers to power a full study. Implementation is the degree to which an intervention can be implemented exactly as proposed. We were particularly interested in examining the utility/participant burden of a battery of assessment and outcome measures for a future trial and also to refine our inclusion/exclusion criteria if necessary. Expansion means a focus on the likely success of an existing evidence-based intervention with a new population or in a novel context. Mindfulness has previously been employed with stroke survivors but not specifically with stroke survivors evidencing low mood. Consequently, we aimed to establish the adequacy of an individualized MBI to provide sufficient motivation for participants to complete the program. Limited-efficacy testing refers to evidence from feasibility studies notwithstanding their inherent limitations such as limited statistical power. We were interested to see if scores on several outcome measures (e.g., mood, quality of life, fatigue) showed positive changes.

\section{Data Analysis}

Data were entered onto an EXCEL spreadsheet and imported to an IBM SPSS version 25 file for statistical analysis. We calculated descriptive statistics (median, mean, SD) for all outcome measures at three timepoints for $n=15$ participants who completed six MBI sessions. We used the non-parametric Wilcoxon Signed Rank Test for comparing T1-T3 scores. In addition, we calculated Cronbach's alphas at T1 $(n=18)$ for all the self-report questionnaires.

\section{Results}

We report the results under five headings based on the general areas of focus for feasibility studies outlined by Bowen et al. (2009). Where participants failed to complete all or most of a questionnaire, this was treated as missing data. Hence, $N$ can vary slightly across measures at any one data collection timepoint (e.g., see Table 4). If a participant only missed a single item within a scale, the mean for that scale was imputed. For example, in the case of our primary outcome measure, the BDI-II, we imputed one item for one participant at time 2 and one item each for two participants at time 3 .

Acceptability Table 2 presents descriptive statistics for both the three eligible participants who completed assessment 1 (baseline) but less than six MBI sessions and the 15 who completed all six sessions. The 15 participants who completed all six sessions were younger than the three who completed less than six, although this difference was not quite significant using the Mann-Whitney $U$ test $(p=0.056)$. They also had higher BDI-II scores, but again, this was not significant $(p=$ $0.07)$ possibly reflecting the small numbers involved. Scores on the LDQ were all very low ranging from 0 to 2 on a scale from 0 to 30 indicating no substance abuse problems.

There were no significant adverse effects from the MBI identified by participants during the MBI, although three reported to the facilitator finding some of the practices challenging because of the emotions that surfaced (e.g., grief), or emotions that they found difficult to achieve (e.g., self-compassion). These effects were managed by the mindfulness facilitator by adjusting the practice, so participants maintained their sense of agency, well-being, and benefit from the programand working with principles of trauma-sensitive mindfulness practice, allowing and supporting people to stay within their window of tolerance (Zhu et al. 2019). 
Table 2 Baseline descriptive statistics for 15 participants completing all six MBI sessions and three participants completing less than six sessions

\begin{tabular}{|c|c|c|}
\hline Variable & $\begin{array}{l}\text { Completed }<6 \\
(n=3)\end{array}$ & $\begin{array}{l}\text { Completed } 6 \text { sessions } \\
(n=15)\end{array}$ \\
\hline Age (mean, SD) & $79.00(5.39)$ & $65.87(12.88)$ \\
\hline \multicolumn{3}{|l|}{ Gender } \\
\hline Male & 2 & 8 \\
\hline Female & 1 & 7 \\
\hline \multicolumn{3}{|l|}{ Ethnicity } \\
\hline European & 2 & 13 \\
\hline Maori & 0 & 1 \\
\hline Other & 1 & 1 \\
\hline \multicolumn{3}{|l|}{ Employment } \\
\hline Working (FT or PT) & 0 & 3 \\
\hline Retired & 3 & 9 \\
\hline Homemaker & 0 & 1 \\
\hline Beneficiary & 0 & 1 \\
\hline Missing & 0 & 1 \\
\hline \multicolumn{3}{|l|}{ Hemisphere? of stroke } \\
\hline Left & 1 & 7 \\
\hline Right & 1 & 7 \\
\hline Unknown & 1 & 1 \\
\hline Length of stay in hospital in days (mean, SD) & $1=8$ days & $15.20(17.42)$ \\
\hline Time since stroke in months & $\begin{array}{l}2=\text { missing data } \\
60.00(46.80)\end{array}$ & $26.33(21.41)$ \\
\hline $\begin{array}{l}\text { WAIS-IV Working memory index (Digit Span, Arithmetic) } \\
\text { (mean, SD)* }\end{array}$ & $84(10.53)$ & $90.61(16.32)^{* * *}$ \\
\hline $\begin{array}{l}\text { WAIS-IV Processing speed index (Coding, Symbol Search) } \\
\text { (mean, SD)* }\end{array}$ & $74(-)^{* *}$ & $79.07(17.64) * * *$ \\
\hline ACS Word Choice Test (mean, SD) & $43.50(3.54)^{* *}$ & $47.00(4.07)$ \\
\hline BDI-II (mean, SD) & $14.00(5.56)$ & $24.73(8.78)$ \\
\hline
\end{tabular}

*WAIS-IV indices are age adjusted scores, ${ }^{*} n=2$ and one missing data, $* * * n=13$ due to missing data
Demand As noted above, using a purposive sampling method, we contacted 43 potential participants resulting in 18 participants commencing and 15 completing the full MBI (see the "Participants" section for details). We initially calculated the correlation between the BDI-II at baseline and postintervention because the strength of the correlation between baseline and follow-up patient reported outcome measures is important for estimating the minimum sample size required to power a randomized controlled trial (Walters et al. 2019). Baseline BDI-II scores presented in Table 3 showed a moderate Pearson's correlation with scores at the end of the 6-week MBI $(r=0.51, p=0.04)$ and at 4-week follow-up $(r=0.41$, $p=0.12)$.

Using the baseline BDI-II mean and SD $(24.73,8.78)$, with a moderately strong correlation between baseline and endpoint BDI-II and an alpha of 0.05, this suggests (assuming the same attrition rate) that a sample size of 36 completed follow-ups per arm or 72 participants in total is needed to detect a minimal clinically important difference of 5.8 (Hiroe et al. 2005) with a power of $90 \%$. In the present study,
15 participants completed all six sessions and the top-up session at 4 weeks. Taken together, this suggests that we would need to contact approximately 163 stroke survivors and provide information regarding a trial to recruit 72 participants who complete all six sessions and the top-up at 4 weeks. One concerning result was the low number of indigenous Māori in this feasibility sample of $18(n=1)$.

Implementation As noted in the "Method" section, the baseline assessment battery comprised a broad range of potential outcome and process measures that could be informative in a future randomized controlled trial. These included self-report questionnaires for depression, anxiety, fatigue, quality of life, substance dependency, and mindfulness. The cognitive measures and substance dependency items were only administered at baseline. The remaining self-report measures were administered at all three assessments. While most participants coped well with the assessment battery, it was clearly too long and taxing at baseline for a number. Several participants complained of fatigue and required breaks to complete the 
Table 3 Descriptive statistics (median, mean, SD) for outcome measures at three timepoints for $n=15$ participants who completed six MBI sessions with Wilcoxon Signed Rank Test comparing $\mathrm{T} 1-\mathrm{T} 3$ scores and Cronbach's alphas at T1 $(n=18)$

\begin{tabular}{llll}
\hline Variable $(\alpha$ at T1) & Time 1 & Time 2 & Time 3 \\
\hline BDI-II $(0.89)$ & $23.00,24.73,(8.78)$ & $6.00,9.33,(10.02)$ & $8.00,10.53,(10.18)^{*}$ \\
$\Delta$ BDI-II & - & $-14.40(9.48)$ & $-13.40(10.55)$ \\
HADS anxiety (0.91) & $10.00,10.27,(5.72)$ & $6.00,6.80,(5.36)$ & $6.00,6.87,(5.20)^{*}$ \\
HADS depression $(0.67)$ & $9.00,9.40,(3.02)$ & $5.00,5.53(4.30)$ & $6.00,6.87(5.20)^{*}$ \\
SSQOL (0.94) & $162,154.07,(31.71)$ & $176.50,176.79,(40.97)$ & $183.50,183.92,(40.95)^{*}$ \\
MFIS $(0.94)$ & $45.00,49.47,(16.11)$ & $38.00,30.86,(15.97)$ & $33.67,(21.05)^{*}$ \\
FFMQ & & & \\
Observing $(0.82)$ & $23.00,23.29,(7.13)$ & $28.00,29.00,(4.53)$ & $27.00,28.38,(5.57)$ \\
Describing (0.72) & $22.00,22.64,(4.34)$ & $25.00,24.73,(5.16)$ & $23.00,24.44,(7.40)$ \\
Act with awareness $(0.90)$ & $24.00,24.14,(7.57)$ & $28.00,27.60,(5.18)$ & $26.00,28.29,(8.01)$ \\
Nonjudging (0.91) & $25.00,27.64,(6.73)$ & $29.00,28.29,(6.91)$ & $26.00,27.60,(7.82)$ \\
Nonreactivity $(0.75)$ & $18.00,17.29,(4.71)$ & $21.00,21.71,(6.45)$ & $25.00,24.07,(5.02)^{* *}$ \\
\hline
\end{tabular}

${ }^{*} p<0.01, * * p<0.05$ assessment; two failed to complete the baseline assessment in full. Of note, the three participants who completed fewer than six MBI sessions each scored low on at least two of the WAIS subtests or failed to complete one or more of the subtests. Calculation of Cronbach's alpha for the 18 participants at baseline shows all the measures had acceptable reliability (i.e., $\geq 0.70$ ) with the exception of the Hospital Anxiety and Depression Scale (HADS) (see Table 3).

Table 4 presents the Pearson correlations between number of sessions completed and the four WAIS-IV subscales and the Word Choice scores with confidence intervals. Inspection of Table 4 suggests that Coding and Word Choice are likely to be the best predictors of participant retention although this needs to be confirmed with a much larger sample. Also, given that 15 out of 18 participants completed all six sessions, and only three out of 15 participants scored below recommended performance validity cut-offs on the Word Choice Test, this result must be treated with caution.

Expansion Of the 18 participants who completed the baseline assessment and the first MBI session, 15 (83\%) went on to complete all six sessions with one completing five, one three and one just two sessions. The same 15 participants who completed all six sessions also all completed the top-up session after 4 weeks. This suggests that the time commitment of $1 \mathrm{~h}$ per week is acceptable and most participants found the one-to-one training format sufficiently motivating to complete the 6 -week program. Table 3 reports descriptive statistics for the main outcome measures at three timepoints for the 15 participants who completed all six MBI sessions.

Limited-Efficacy Testing While the present study was primarily intended to provide data to calculate the minimal sample size to power a robust trial, we have also reported descriptive statistics at all three time periods for all main outcome measures in Table 3. Non-parametric MannWhitney $U$ tests show significant within-subject changes across all measures reflecting improved well-being. While this was a feasibility study and not powered to detect real changes, it is worth noting that mean BDI-II scores improved by $>1$ standard deviation, and this was maintained at follow-up. Moreover, inspection of individual change scores on the BDI-II (see Table 3 for descriptive statistics) showed these were larger than the MCID (i.e., 5.8) for 14 participants with a single participant whose BDI-II score increased. Anxiety on the Hospital Anxiety and Depression Scale reduced significantly between baseline and at the end of the MBI and this was maintained at follow-up.
Table 4 Pearson correlations with confidence intervals between WAIS-IV subscale and Word Choice raw scores and number of sessions completed

\begin{tabular}{lllllc}
\hline & $\begin{array}{l}\text { Digit span } \\
(n=17)\end{array}$ & $\begin{array}{l}\text { Arithmetic } \\
(n=17)\end{array}$ & $\begin{array}{l}\text { Symbol search } \\
(n=15)\end{array}$ & $\begin{array}{l}\text { Coding } \\
(n=17)\end{array}$ & $\begin{array}{l}\text { Word choice } \\
(n=16)\end{array}$ \\
\hline$r=$ & 0.09 & 0.28 & 0.29 & 0.38 & 0.36 \\
$95 \%$ CI & -0.41 to 0.55 & -0.23 to 0.67 & -0.26 to 0.7 & -0.12 to 0.73 & -0.17 to 0.73 \\
\hline
\end{tabular}




\section{Discussion}

We provided 6 weeks of a one-to-one MBI to 18 stroke survivors to examine feasibility issues which needed clarification before a randomized controlled trial could be considered. In particular, we examined acceptability, demand, implementation, expansion, and limited-efficacy testing with evidence across all five of these criteria for the feasibility of MBIs with stroke survivors with low mood. Overall, participants found the time commitment, weekly sessions, and 60-min session duration were acceptable with 15 of 18 participants completing all six sessions and the top-up or booster session 4 weeks later. These recruitment data enable precise calculation of numbers needed to power a randomized controlled trial. However, the cognitive tests included at baseline were demanding for some participants and the choice of cognitive measures would need to be carefully considered in any proposed trial. Improved mood and well-being were evident across outcome measures at the end of the MBI and at follow-up 4 weeks later. Adverse effects were not reported although some participants found some aspects of the MBI challenging especially where there was a history of trauma. These results indicate that a controlled trial of a MBI for improving mood in stroke survivors is feasible and should be pursued.

The time commitment, format, and content of the training provided were acceptable and well-received, with 15 out of 18 participants completing all six sessions plus a top-up session 4 weeks later. Interestingly, the three participants who completed some sessions, but not all six sessions, were typically older and less depressed (at baseline) than the 15 who completed all six sessions. While these differences were not significant, possibly due to the small number involved, it could suggest that younger participants with lower mood have more incentive to attend all sessions.

While the present study did not have a control group and was not powered to establish significant effects, it is worth noting that the mean BDI-II score at baseline decreased after six sessions by about 1.5 standard deviations and this improvement was maintained at four-week follow-up. At an individual level, this was reflected by changes in the BDI-II between baseline and 4-week follow-up that were larger than the MCID for 14 of the 15 participants who completed the six sessions. Moreover, the improved depression scores were accompanied by positive shifts in anxiety, quality of life, and mindfulness. These results provide provisional support for conducting a large trial of a MBI for improving low mood in stroke survivors.

In addition to the positive aspects of the MBI, there was little evidence of any harmful or major side effects. At the same time, three participants reported experiencing some emotional discomfort on occasion or being emotionally challenged by some of the practices. These effects were managed by the mindfulness facilitator by adjusting the practice, so participants maintained their sense of agency and learnt to make mindful choices that supported their well-being and enabled them to benefit from the program overall. This highlighted the need for the therapist delivering the intervention to be experienced and able to adapt the program to meet these individual needs while maintaining program integrity. In the present study, we had no information about participants' personal history in terms of trauma, so the MBI had to "hold" this space sensitively and carefully while participants were learning to navigate this potentially challenging terrain. For a future study, it may be useful if not important to include a questionnaire in the selection process that may identify any past trauma. In addition, it is important to alert MBI participants to the possibility of such experiences occurring and to discuss in advance the best ways of managing them.

At the same time, these experiences reported by participants should not necessarily be interpreted as negative side effects or as undesirable. Indeed, an important part of a MBI is learning to acknowledge, accept, and be present with physical pain and/or emotional distress. Baer et al. (2019) note that MBI programs often include an exercise that involves asking participants to think of a problem or distressing experience and to observe the feelings and sensations this elicits with a "friendly curiosity" (p.106). They make the point that most psychotherapy and physical exercise interventions typically require some degree of mental or physical discomfort in order to achieve improved functioning and well-being. The reader is referred to their recent review for a more in-depth account of adverse effects of meditation (Baer et al. 2019). Interestingly, they suggest that harmful effects of behavioral interventions (e.g., psychotherapy, exercise) can be considered in terms of three aspects, namely: (i) the characteristics of the program, (ii) the participant, and (iii) the teacher/clinician. For a detailed discussion of adverse effects, from both a traditional Buddhist and a contemporary mindfulness perspective, we refer the reader to the paper by Anālayo (2019).

In the present study, we employed an experienced mindfulness facilitator with a professional background in occupational therapy, including working with stroke survivors, who was able to skillfully manage any reported distress in participants. Nonetheless, we concur with Baer et al. (2019) that any trials of MBIs must actively monitor adverse effects among individuals since group averages can show overall improvement while concealing individual scores indicative of serious deterioration. In most previous studies of MBIs for people with neurological conditions, this issue has been ignored (Siegert et al. 2015). We also suggest that any MBI available for stroke survivors needs to be trauma informed (Zhu et al. 2019).

One important finding from this study was that our baseline assessment battery was too demanding or burdensome for some participants. This was evident in both their subjective 
comments and missing data when participants failed to complete a measure. It was also apparent that it was primarily the cognitive tests that people found most taxing. The aim of including these cognitive tasks was to examine their feasibility for inclusion in a larger trial: first, as tests that might identify participants who are too cognitively impaired to undertake or benefit from a MBI; second, as tests that might be useful as statistical predictors of participant retention. In the present study, the WAIS-IV Symbol Search subtest and Advanced Clinical Solutions Word Choice Test had the highest correlations with the number of sessions completed. These are likely to be the two most useful cognitive tests from our battery for screening out participants who might struggle to complete at least six sessions of a MBI. However, this correlational relationship was based on a small sample in which most participants completed all sessions - and should be replicated in a much larger sample.

Another important feasibility issue that we investigated was the number of potential participants we would have to recruit to power a trial. Notwithstanding the moderately strong correlation observed between the primary outcome (BDI-II) at baseline and the endpoint (i.e., four-week follow-up), this still required finding 163 stroke survivors to recruit 72 participants sufficient to power a trial assuming the same attrition rate as for the present study. In other words, it would be necessary to find and communicate with slightly more than two stroke survivors for every trial participant recruited. This ratio might be improved with a more focused recruitment strategy since $17 / 43$ of the non-participants did not meet the inclusion criteria.

One concern arising from the results of the present study was the low proportion of Māori, the indigenous people of New Zealand, participating - only one participant out of 18 . The 2018 New Zealand census reported that Māori comprised $16.5 \%$ of the population up from $14.9 \%$ in 2013 (Stats NZ 2018). It may be necessary in future research to attempt to employ "maximum variation sampling" to obtain proportionate representation. Furthermore, touchpoints and the relevance of the Māori world view of mindfulness including Aio (harmony, at peace, calm), Inaiāne (now, present time), and Wairua (spirit of a person in life and after death), and how to adapt and align with wider research in mindfulness, are also deserving of further attention from mindfulness practitioners and researchers.

\section{Limitations and Future Research}

The present study is limited by a small sample size, the lack of a randomized control group, and the absence of long-term follow-up. Moreover, the MBI was delivered individually and it is uncertain how this compares with the standard or traditional delivery in a weekly group setting. This study is also limited by the possible effects of common method bias due to the reliance upon self-report questionnaires as outcome measures (Podsakoff et al. 2003). There is considerable evidence from a range of disciplines that common method variance can either inflate or deflate the relationships observed among constructs. Consequently, it is important that any future trial of MBIs should endeavor to use more diverse outcome measure modalities. Given these limitations, it is important not to regard the present study as evidence for the effectiveness of MBIs for improving mood in stroke survivors.

Notwithstanding these limitations, we found that MBI training delivered individually over six weekly sessions was acceptable to stroke survivors with low mood, with most participants demonstrating improved scores on measures of depression, anxiety, and stroke specific quality of life. Three participants reported feeling emotionally challenged by some of the practices and we recommend that MBIs for stroke survivors be provided by practitioners experienced in mindfulness, working with stroke survivors and trauma-informed therapy. Importantly, this study established the feasibility of providing a MBI for stroke survivors who have low mood. It is important now to conduct rigorous randomized controlled trials to test the effectiveness and efficacy of MBIs for enhancing mood in stroke survivors.

\section{Ethics}

Ethical approval to carry out this study was obtained from the Northern Area Health and Disability Ethics Committee (approval number 17/NTA/147) and Auckland University of Technology's Ethics Committee (approval number 17/262). Informed written consent was obtained prior to or at the first assessment.

Supplementary Information The online version contains supplementary material available at https://doi.org/10.1007/s12671-020-01583-4.

Acknowledgments We would like to thank the Stroke Foundation New Zealand and its regional stroke coordinators for assistance with recruiting participants and all of our participants for their time and contribution to the study. Alice Theadom was supported by a Rutherford Discovery Fellowship, administered by The Royal Society of New Zealand.

Authors' Contributions RS designed and led the study, completed the statistical analyses, and co-wrote the first draft. WW collaborated in the design of the study and project management and co-wrote the first draft. MD provided the MBI and collaborated in design and writing up. JW managed all operational aspects of recruitment and data collection and collaborated in writing up. AT collaborated in the design of the study, project oversight, and writing up. NK collaborated in the design of the study, project oversight, and writing up. DS collaborated in the design of the study, advised on cognitive testing, provided project oversight, and contributed to writing up. SR collaborated in the design of the study, project oversight, and writing up. MR advised on cultural issues, the design of the study, project oversight, and writing up. DB collaborated in the design of the study, project oversight, and writing up. ST collaborated in the design of the study and advised on statistics. 
Funding This work was supported by the Health Research Council of New Zealand under grant 17/522.

\section{Compliance with Ethical Standards}

Conflict of Interest The authors declare that they have no conflict of interest.

Open Access This article is licensed under a Creative Commons Attribution 4.0 International License, which permits use, sharing, adaptation, distribution and reproduction in any medium or format, as long as you give appropriate credit to the original author(s) and the source, provide a link to the Creative Commons licence, and indicate if changes were made. The images or other third party material in this article are included in the article's Creative Commons licence, unless indicated otherwise in a credit line to the material. If material is not included in the article's Creative Commons licence and your intended use is not permitted by statutory regulation or exceeds the permitted use, you will need to obtain permission directly from the copyright holder. To view a copy of this licence, visit http://creativecommons.org/licenses/by/4.0/.

\section{References}

Alsubaiea, M., Abbott, R., Dunna, B., Dickens, C., Keil, T. F., Henley, W., \& Kuyken, W. (2017). Mechanisms of action in mindfulnessbased cognitive therapy (MBCT) and mindfulness-based stress reduction (MBSR) in people with physical and/or psychological conditions: a systematic review. Clinical Psychology Review, 55, 74 91.

Anālayo, B. (2019). The insight knowledge of fear and adverse effects of mindfulness practices. Mindfulness, 10, 2172-2185. https://doi.org/ 10.1007/s12671-019-01198-4.

Anderson, C. S., Carter, K. N., Brownlee, W. J., Hackett, M. L., Broad, J. B., \& Bonita, R. (2004). Very long-term outcome after stroke in Auckland, New Zealand. Stroke, 35(8), 1920-1924. https://doi. org/10.1161/01.Str.0000133130.20322.9f.

Baer, R., Crane, C., Miller, E., \& Kuyken, W. (2019). Doing no harm in mindfulness-based programs: conceptual issues and empirical findings. Clinical Psychology Review, 71, 101-114.

Bain, K. M., \& Soble, J. R. (2019). Validation of the advanced clinical solutions word choice test (WCT) in a mixed clinical sample: establishing classification accuracy, sensitivity/specificity, and cutoff scores. Assessment, 26(7), 1320-1328.

Beck, A. T., Steer, R. A., \& Brown, G. K. (1996). Beck depression inventory and Manual (2nd ed.). San Antonio, TX: Psychological Corporation.

Bowen, D. J., Kreuter, M., Spring, B., Cofta-Woerpel, L., Linnan, L., Weiner, D., Bakken, S., Kaplan, C. P., Squiers, L., Fabrizio, C., \& Fernandez, M. (2009). How we design feasibility studies. American Journal of Preventive Medicine, 36(5), 452-457.

Burton, C. A. C., Holmes, J., Murray, J., Gillespie, D., Lightbody, C. E., Watkins, C. L., \& Knapp, P. (2011). Interventions for treating anxiety after stroke. Cochrane Database of Systematic Reviews, 12. https://doi.org/10.1002/146518.CD008860.pub2.

Cumming, T. B., Blomstrand, C., Skoog, I., \& Linden, T. (2016). The high prevalence of anxiety disorders after stroke. American Journal of Geriatric Psychiatry, 24(2), 154-160. https://doi.org/10.1016/j. jagp.2015.06.003.

de Abreu Costa, M., D'Alò de Oliveira, G. S., Tatton-Ramos, T., Manfro, G. G., \& Salum, G. A. (2019). Anxiety and stress-related disorders and mindfulness-based interventions: a systematic review and multilevel meta-analysis and meta-regression of multiple outcomes.
Mindfulness, 10, 996-1005. https://doi.org/10.1007/s12671-0181058-1.

Djukanovic, I., Carlsson, J., \& Årestedt, K. (2017). Is the Hospital Anxiety and Depression Scale (HADS) a valid measure in a general population 65-80 years old? A psychometric evaluation study. Health and Quality of Life Outcomes, 15, 193. https://doi.org/10. 1186/s12955-017-0759-9.

Farias, M., Maraldi, E., Wallenkampf, K. C., \& Lucchetti, G. (2020). Adverse events in meditation practices and meditation-based therapies: a systematic review. Acta Psychiatrica Scandinavica, 1-19. https://doi.org/10.1111/acps.13225.

Gu, J., Strauss, C., Crane, C., Barnhofer, T., Karl, A., Cavanagh, K., \& Kuyken, W. (2016). Examining the factor structure of the 39-item and 15 -item versions of the five facet mindfulness questionnaire before and after mindfulness-based cognitive therapy for people with recurrent depression. Psychological Assessment, 28(7), 791-802.

Hackett, M. L., \& Pickles, K. (2014). The frequency of depression after stroke: an updated systematic review and meta-analysis of observational studies. International Journal of Stroke, 8, 1017-1025.

Hackett, M. L., Anderson, C. S., House, A. O., \& Xia, J. (2009). Interventions for treating depression after stroke. Stroke, 40(7), E487-E488. https://doi.org/10.1161/Strokeaha.109.547059.

Hiroe, T., Kojima, M., Yamamoto, I., Nojima, S., Kinoshita, Y., Hashimoto, N., Watanabe, N., Maeda, T., \& Furukawa, T. A. (2005). Gradations of clinical severity and sensitivity to change assessed with the Beck Depression Inventory-II in Japanese patients with depression. Psychiatry Research, 135(3), 229-235. https://doi. org/10.1016/j.psychres.2004.03.014.

Hoffmann, T. C., Glasziou, P. P., Boutron, I., Milne, R., Perera, R., Moher, D., Altman, D. G., Barbour, V., Macdonald, H., Johnston, M., Lamb, S. E., Dixon-Woods, M., McCulloch, P., Wyatt, J. C., Chan, A., \& Michie, S. (2014). Better reporting of interventions: template for intervention description and replication (TIDieR) checklist and guide. BMJ [British Medical Journal], 348, ARTN g1687. https://doi.org/10.1136/bmj.g1687.

Jani, B. D., Simpson, R., Lawrence, M., Simpson, S., \& Mercer, S. W. (2018). Acceptability of mindfulness from the perspective of stroke survivors and caregivers: a qualitative study. Pilot and Feasibility Studies, 4. https://doi.org/10.1186/s40814-018-0244-1.

Jaseja, J. (2006). A brief study of a possible relation of epilepsy association with meditation. Medical Hypotheses, 66(5), 1036-1037.

Kelly, J. F., Magill, M., Slaymaker, V., \& Kahler, C. (2010). Psychometric validation of the Leeds Dependence Questionnaire (LDQ) in a young adult clinical sample. Addictive Behaviors, 35(4), 331-336. https://doi.org/10.1016/j.addbeh.2009.11.005.

Kos, D., Kerckhofs, E., Carrea, I., Verza, R., Ramos, M., \& Jansa, J. (2005). Evaluation of the Modified Fatigue Impact Scale in four different European countries. Multiple Sclerosis, 11, 76-80.

Lawrence, M., Booth, J., Mercer, S., \& Crawford, E. (2013). A systematic review of the benefits of mindfulness-based interventions following transient ischemic attack and stroke. International Journal of Stroke, 8(6), 465-474. https://doi.org/10.1111/ijs.12135.

Lazarus, A. A. (1976). Psychiatric problems precipitated by Transcendental Meditation. Psychological Reports, 39, 601-602.

Lichtenberger, E. O., \& Kaufman, A. S. (2009). Essentials of WAIS-IV assessment. Hoboken: John Wiley \& Sons, Inc..

Muus, I., Williams, L. S., \& Ringsberg, K. C. (2007). Validation of the Stroke Specific Quality of Life Scale (SS-QOL): test of reliability and validity of the Danish version (SS-QOL-DK). Clinical Rehabilitation, 21, 620-627.

Pearson. (2009). Advanced clinical solutions for WAIS-IVC and WMS$I V($ C. Clinical and Interpretive Manual. San Antonio: Pearson.

Podsakoff, P. M., MacKenzie, S. B., Lee, J. Y., \& Podsakoff, N. P. (2003). Common method biases in behavioral research: a critical review of the literature and recommended remedies. Journal of Applied Psychology, 88(5), 879-903. 
Raistrick, D., Bradshaw, J., Tober, G., Weiner, J., Allison, J., \& Healey, C. (1994). Development of the Leeds Dependence Questionnaire (LDQ): a questionnaire to measure alcohol and opiate dependence in the context of a treatment evaluation package. Addiction, 89(5), $563-572$.

Siegert, R. J., Rowland, V., \& Theadom, A. (2015). Critical review of mindfulness interventions for neurological conditions. Presented at the Australasian Federation of Rehabilitation Medicine/New Zealand Rehabilitation Association Joint Meeting, 13-17 October 2015, Wellington, NZ.

Stats, N. Z. (2018). New Zealand Census. Retrieved from https://www. stats.govet.nz/2018-census/. Accessed 14 June 2020.

Taylor, W. J., Wong, A., Siegert, R. J., \& McNaughton, H. K. (2006). Effectiveness of a clinical pathway for acute stroke care in a district general hospital: an audit. BMC Health Services Research, 6. https:// doi.org/10.1186/1472-6963-6-16.

Thomas, R., Chur-Hansen, A., \& Turner, M. (2020). A systematic review of studies on the use of Mindfulness-Based Cognitive Therapy for the treatment of anxiety and depression in older people. Mindfulness, 11, 1599-1609. https://doi.org/10.1007/s12671-02001336-3.

Walker, E. R., Obolensky, N., Dini, S., \& Thompson, N. J. (2010). Formative and process evaluations of a cognitive-behavioral therapy and mindfulness intervention for people with epilepsy and depression. Epilepsy \& Behavior, 19(3), 239-246. https://doi.org/10.1016/ j.yebeh.2010.07.032.

Walters, S. J., Jacques, R. M., Henriques-Cadby, I. B. d. A., Candlish, J., Totton, N., \& Xian, M. T. S. (2019). Sample size estimation for randomized controlled trials with repeated assessment of patient- reported outcomes: what correlation between baseline and followup outcomes should we assume? Trials, 20, 566. https://doi.org/10. 1186/s13063-019-3671-2.

Wang, M., Liao, W., \& Chen, X. (2019a). Effects of a short-term mindfulness-based intervention on comfort of stroke survivors undergoing inpatient rehabilitation. Rehabilitation Nursing, 44, 78-86.

Wang, X., Smith, C., Ashley, L., \& Hyland, M. E. (2019b). Tailoring self-help mindfulness and relaxation techniques for stroke survivors: examining preferences, feasibility and acceptability. Frontiers in Psychology, 10, 391. https://doi.org/10.3389/fpsyg.2019.00391.

Wechsler, D. (2008). Wechsler adult intelligence scale - fourth edition (WAIS-IV). San Antonio: Pearson.

Williams, L. S., Weinberger, M., Harris, L. E., Clark, D. O., \& Biller, J. (1999). Development of a stroke specific quality of life scale. Stroke: A Journal of Cerebral Circulation, 30(7), 1362-1369.

Wood, K., Lawrence, M., Jani, B., Simpson, R., \& Mercer, S. W. (2017). Mindfulness-based interventions in epilepsy: a systematic review. BMC Neurology, 17, 52. https://doi.org/10.1186/s12883-017-0832-3.

Zhu, J., Wekerle, C., Lanius, R., \& Frewen, P. (2019). Trauma- and stressor-related history and symptoms predict distress experienced during a brief mindfulness meditation sitting: moving toward trauma-informed care in mindfulness-based therapy. Mindfulness, 10, 1985-1996.

Zigmond, A. S., \& Snaith, A. P. (1983). The hospital anxiety and depression scale. Acta Psychiatrica Scandinavica, 67(6), 361-370.

Publisher's Note Springer Nature remains neutral with regard to jurisdictional claims in published maps and institutional affiliations. 